\title{
Parametric Solitons in Nonlinear Photonic Crystals
}

\author{
Katia Gallo \\ Optoelectronics Research Centre, University of Southampton, Southampton SO17 1BJ, United Kingdom \\ Salvatore Stivala, Alessia Pasquazi and Gaetano Assanto \\ Nonlinear Optics and Optoelectronics Lab, University "Roma Tre", Via della Vasca Navale 84, 00146 Rome, Italy
}

\begin{abstract}
We present theoretical and experimental investigations on the soliton dynamics associated to multiple second harmonic generation resonances in two-dimensional nonlinear photonic crystals, highlighting a wealth of new possibilities for soliton management in such structures.
\end{abstract}

Self-guiding filaments of light or optical spatial solitons, resulting from the balance between nonlinearity and diffraction, hold promise for the development of novel, reconfigurable photonic architectures for switching and routing. They have been predicted and demonstrated in a variety of physical settings [1], including quadratic media. In the latter case, diffractive beam spreading is counteracted by the parametric interplay of three wavelength components, resulting in the mutual trapping and locking of multi-frequency waves (i. e. multicolor solitons). The original predictions on soliton formation via three-wave mixing [2] were confirmed nearly a decade ago by the first experiments carried out in KTP and $\mathrm{LiNbO}_{3}$ [3-4]. Since then, the development of Quasi-Phase-Matching (QPM) materials such as Periodically Poled $\mathrm{LiNbO}_{3}$ (PPLN) has opened up new possibilities for quadratic soliton science and engineering [5-6]. QPM has also recently been extended to higher-dimensionalities, to demonstrate 2D PPLN Nonlinear Photonic Crystals (NPC), i.e. 2D lattices in the second-order susceptibility $\chi^{(2)}$, enabling novel and more versatile geometries for parametric interactions [7-8].

Here we report the first experimental and theoretical investigations on soliton formation in two-dimensional NPC, using hexagonal lattices in lithium niobate. The studies were performed in a $(1+1) \mathrm{D}$ configuration, using buried planar waveguides embedded in the NPC structure for maximum efficiency [9]. The results unveil an excitingly rich scenario for soliton physics and optical processing, arising as a result of multiple spatial - as well as spectral nonlinear resonances in the $2 \mathrm{D}$ nonlinear lattice.

The 2D NPC used in the experiments is an Hexagonally Poled Lithium Niobate (HexLN) planar waveguide fabricated by reverse-annealed proton-exchange [9], optimised for twin-beam second harmonic generation (TB SHG) from $\lambda_{\omega} \sim 1.55 \mu \mathrm{m}$ (Fig. 1).

The 2D nonlinear lattice exhibits two second harmonic generation resonances, $\lambda_{\omega}=\lambda_{1}$ and $\lambda_{\omega}=\lambda_{2}$, the spectral location of which can be adjusted around the degeneracy point $\left(\lambda_{\omega}=\lambda_{1} \equiv \lambda_{2}\right)$ by tuning the incidence angle $\left(\theta_{\omega}\right)$ of the pump guided in the sample, as shown in Fig. $1 b$.

a)

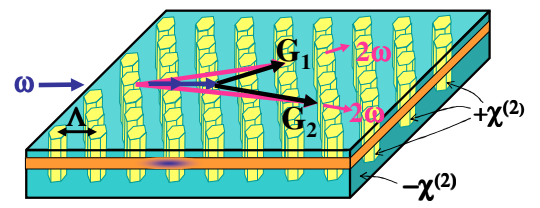

b)

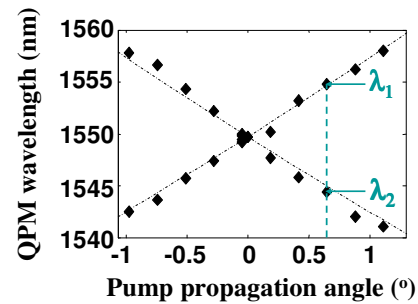

Fig. 1: $a$ ) Sketch of the 2D HexLN waveguide (period: $\Lambda=16.4 \mu \mathrm{m}$ ) used for twin-beam SHG; $b$ ) variation of the two SHG wavelengths: $\lambda_{1}$ and $\lambda_{2}$, with the pump propagation angle.

Soliton formation was numerically investigated through the following set of coupled-mode equations, written in terms of the slowly varying envelopes of the fundamental (FF: $A$ ) and second harmonics ( $\mathrm{SH}: B_{1}, B_{2}$ ), with normalised longitudinal and transverse coordinates: $\zeta=x / \mathrm{L}_{\mathrm{D}}$ and $\xi=y / w_{0}$ ( $w_{0}$ being the FF input beam waist):

$$
\begin{aligned}
& \frac{\partial A}{\partial \zeta}+i \sigma \frac{\partial^{2} A}{\partial \xi^{2}}=-i \Gamma_{1} A^{*} B_{1}-i \Gamma_{2} A^{*} B_{2} \\
& \frac{\partial B_{1}}{\partial \zeta}+\rho_{1} \frac{\partial B_{1}}{\partial \xi}+i \sigma_{1} \frac{\partial^{2} B_{1}}{\partial \xi^{2}}+i \delta \beta_{1} B_{1}=-i \Gamma_{1} A^{2} \\
& \frac{\partial B_{2}}{\partial \zeta}+\rho_{2} \frac{\partial B_{2}}{\partial \xi}+i \sigma_{2} \frac{\partial^{2} B_{2}}{\partial \xi^{2}}+i \delta \beta_{2} B_{2}=-i \Gamma_{2} A^{2}
\end{aligned}
$$

where $\Gamma_{1,2}$ and $\delta \beta_{1,2}\left\{\delta \beta_{1,2}=\left[\beta_{1,2}(2 \omega)-2 \beta(\omega)-G_{1,2}\right] L_{D}, L_{D}\right.$ being the FF diffraction length in the sample $\}$ are the normalised SHG coupling coefficients and mismatches, respectively. $\sigma=1 / 4$ and $\sigma_{1,2} \sim 1 / 8$ account for diffraction at the FF and the SH and $\rho_{1,2} \sim \pm 1.5$ for the in-plane angular deviations of the SH beams. 
At higher excitations $\left(\Gamma_{1,2}\right)$ the two SHG resonances tend to lock the FF wave to the SH yielding a nonspreading multicolour filament of light, i.e. a parametric spatial soliton. The additional in-plane degrees of freedom of the nonlinear lattice yield substantially different results from the 1D SHG soliton case, with an enhanced range for FF wave confinement (in the regions of positive $\delta \beta$ 's) and opposite beam displacements with respect to the input direction, controlled by the interplay of $\delta \beta_{1}$ and $\delta \beta_{2}$.

We verified these predictions through nonlinear experiments in the planar HexLN configuration of Fig. 1, using a narrow-linewidth, pulsed $(20 \mathrm{ps})$ infrared source tunable around $1550 \mathrm{~nm}$, with input focusing conditions corresponding to propagation over more than five diffraction lengths in the NPC. For a quantitative study of selfconfinement we monitored the evolution of the FF spot-size ( $\left.w_{\text {out }}\right)$ and lateral displacement $\left(\Delta y_{\text {out }}\right)$ at the crystal endfacet as a function of the three main control parameters: FF launch peak-power $\left(\mathrm{P}_{\omega}\right)$, wavelength $\left(\lambda_{\omega}\right)$ and angle of propagation $\left(\theta_{\omega}\right)$. For pump wavelengths close to $\lambda_{1}$ and $\lambda_{2}$ we could observe self-confinement of the FF beam to lateral widths comparable to the input at (external) input peak powers $P_{\omega} \geq 20 \mathrm{~kW}$. The sample yielded an extremely rich soliton dynamics and different soliton behaviours when moving from degeneracy for coincident resonances [i.e. $\left.\lambda_{1} \equiv \lambda_{2} \rightarrow \delta \beta_{1} \equiv \delta \beta_{2} \equiv \delta \beta\left(\lambda_{\omega}\right)\right]$ in the symmetric TB SHG case, to fully decoupled SHG processes in the asymmetric case. Results for the latter are illustrated by Fig.2, where two spectral regions sustaining beam confinement with opposite displacements can be clearly identified just after the resonant wavelengths $\left(\lambda_{1}\right.$ and $\left.\lambda_{2}\right)$. This enables light routing by acting on the input wavelength $\left(\lambda_{\omega}\right)$, an entirely new approach to soliton steering.
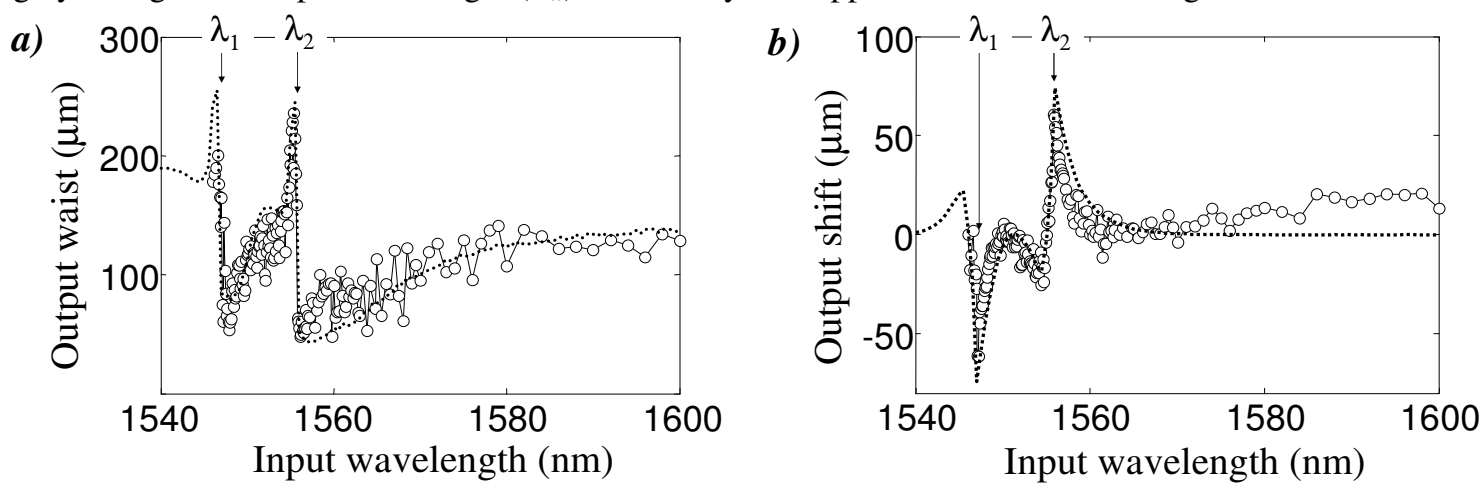

Fig. 2: Asymmetric (non degenerate) twin beam-SHG, with $\theta_{\omega}=0.58^{\circ}, \lambda_{1}=1546.5 \mathrm{~nm}, \lambda_{2}=1555.7 \mathrm{~nm}$. Measurements of $a$ ) the beamwaist and $b$ ) the lateral shift of the output FF beam, for $\mathrm{P}_{\omega}=22 \mathrm{~kW}$ (dots). The dotted lines show predictions from model (1).

In conclusion, simulations and experiments on doubly-resonant SHG reveal the possibility to counterbalance linear diffraction via quadratic nonlinearities to generate a novel class of parametric solitary waves in 2D nonlinear photonic crystals. Preliminary results highlight intriguing features in the wave dynamics arising from the excitation of multiple nonlinear resonances in the 2D lattice. In a HexLN planar waveguide we demonstrated the existence of two spectral regions for beam confinement and opposite displacements controllable with the input FF wavelength, angle and power. We anticipate these findings to be a starting point for wave dynamics engineering in a variety of two- and three-dimensional nonlinear photonic crystals, aperiodic lattices and quasi-crystals.

Katia Gallo gratefully acknowledges support from the Leverhulme Trust (Special Fellowship SRF/40112). The other authors thank Dr. L. Colace for discussions and Italian MIUR (PrIN nr. 2005098337) for partial funding.

[1] Y. S. Kivshar and G. P. Agrawal, Optical Solitons: from fibres to photonic crystals, Academic, New York, 2003.

[2] Y. N. Karamzin and A. P. Sukhorukov, Mutual focusing of high-power light beams in media with quadratic nonlinearity, Sov. Phys. JETP 41, 414 (1976).

[3] W. E. Torruellas et al., Observation of two-dimensional spatial solitary waves in a quadratic medium, Phys. Rev. Lett. 74, 5036 (1995).

[4] R. Schiek, Y. Baek, G. I. Stegeman, One-dimensional spatial solitary waves due to cascaded second-order nonlinearities in planar waveguides, Phys. Rev. E 53, 1138 (1996).

[5] B. Bourliaguet et al., Observation of quadratic spatial solitons in periodically poled lithium niobate, Opt. Lett. 24, 1410 (1999).

[6] R. Schiek et al., One-dimensional spatial soliton families in optimally engineered quasi-phase-matched lithium niobate waveguides, $O p t$. Lett. 29, 596 (2004).

[7] V. Berger, Nonlinear Photonic Crystals, Phys. Rev. Lett. 81, 4136 (1998).

[8] N. G. R. Broderick et al., Hexagonally poled lithium niobate: a two-dimensional nonlinear photonic crystal, Phys. Rev. Lett. 84, 4345 (2000).

[9] K. Gallo et al., Guided-wave second harmonic generation in a $\mathrm{LiNbO}_{3}$ nonlinear photonic crystal, Opt. Lett. 31, 1232 (2006). 